\title{
A Comparative Study on Extra-Corporal Circulation Control
}

\author{
Benedikt Baumgartner, Alejandro Mendoza, Stefan Eichhorn, Ulrich Schreiber and Alois Knoll
}

\begin{abstract}
Hydraulic models of the human circulatory system allow simulation of hemodynamics in a standardized setting. In this work we use such a model to compare concurrent control strategies for extra-corporal circulation control. Patients suffering from cardio-vascular diseases under extra-corporal circulation can benefit from an automated regulation. The control task is to keep either the pump flow or the pressure in the cardio-vascular system at a constant target value, despite any parameter variations or external perturbations. We implement four control strategies that seem favorable for our task: a Proportional-Integral Controller, a $H_{\infty}$-Controller, a PIFuzzy Controller and a Model Reference Adaptive Controller. The methods are compared quantitatively and their advantages and drawbacks are discussed.
\end{abstract}

\section{INTRODUCTION}

Heart-lung machines (HLM) are used in cardiac surgery on a daily basis and are operated by trained perfusionists. An automated extra-corporal circulation (ECC) can help to increase patient safety and to reduce the workload of the perfusionist. In this study we setup a hydraulic circulatory model and evaluate 4 control strategies for automated ECC control.

Cardio-vascular system (CVS) modelling has been subject to numerous studies. One of the most popular artificial models is the 3-element windkessel or Westerhof model, introduced in 1971 [1]. Some years later Stergiopulos et al. [2] presented a fourth element (inductivity) to improve accuracy in estimating total arterial compliance. Recently, Kind et al. [3] questioned the use of a four-element model, due to low identifiability of the inductivity. Also Sharp and Dharmalingam find the three-element model among others to exhibit the best tradeoff between simplicity and accuracy [4].

Also ECC control has been adressed in numerous publications. Back in 1978 Prilutskii et al. considered basic design principles for an automated ECC [5]. Rao et al. developed a model predictive controller to regulate hemodynamic variables in critical care subjects and revealed that automatic control outperformed manual regulation [6]. Misgeld et al. compared three different control schemes to regulate blood flow [7]: A proportional-integral (PI) controller, a selftuning general predictive controller (GPC) and a $H_{\infty}$-Controller, which showed the best control performance during tests in a

Benedikt Baumgartner, Alejandro Mendoza and Alois Knoll are with the Robotics and Embedded Systems Group, Department of Computer Science, Technische Universitaet Muenchen, Boltzmannstr. 3, 85748 Garching b. Muenchen, Germany, \{baumgarb, mendozag, knoll\}ein.tum.de.

Stefan Eichhorn and Ulrich Schreiber are with the German Heart Center Munich, Lazarettstr. 36, 80636 Muenchen, Germany, \{stefan.eichhorn, schreiberu\}@dhm.mhn.de. circulatory simulator. Meyrowitz developed a prototype of an automatically controlled heart-lung machine using PID and predictive controllers [8].

Based on these studies we implement a PI-Controller, a $H_{\infty}$ Controller, a Model Reference Adaptive Controller and a PIFuzzy Controller. We designed the controllers robustly and compare their performance.

\section{METHODS}

\section{A. Hydraulic Circulatory Model}

1) Layout: For a mechanical model of the circulatory system we follow the Westerhof model. It consists of two resistances (R) and a compliance element (C) in a RCR configuration, representing characteristic arterial impedance, arterial compliance and peripheral resistance respectively [4]. The HLM is represented by a motor-driven centrifugal pump, connected to a venous and arterial cannula at its in- and outlet. Figure 1 shows a schematic overview of our setup. Several sensors are used to measure the model's performance. Two pressure sensors measure the in- and outlet of the CVS component. A flow sensor and a tachometer observe pump parameters. An AD/DA-Converter together with a control unit (laptop) is used to receive sensor signals as well as to control the motor speed and the variable resistances of the patient module. In order to design controllers for the

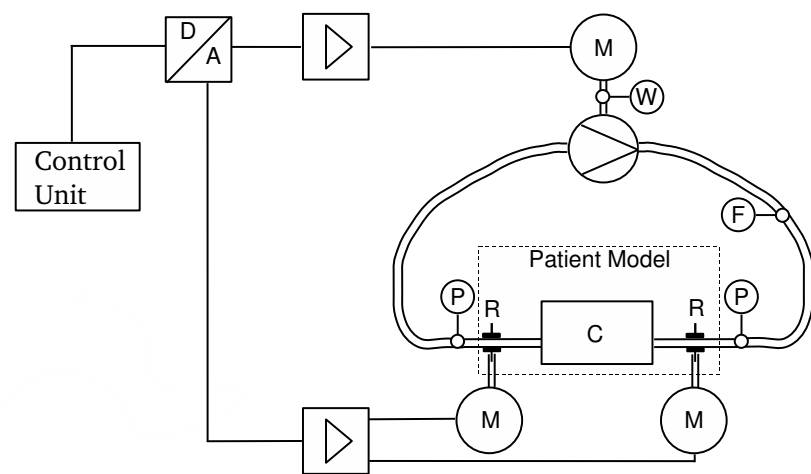

Fig. 1. Schematic layout of the hydraulic mock model with connected sensors and control unit.

plant, an analytical description of the components is derived in the following sections.

2) Motor: To drive the centrifugal pump we use a direct current motor. The motor speed is measured by a tachometer. A mathematical representation of the motor follows the state 
space model described in [9]:

$$
\begin{aligned}
{\left[\begin{array}{c}
\dot{I}_{A}(t) \\
\dot{\omega}(t)
\end{array}\right]=} & {\left[\begin{array}{cc}
-\frac{R_{A}}{L_{A}} & -\frac{K_{T}}{L_{A}} \\
\frac{K_{T}}{J} & -\frac{T_{F l}}{J}
\end{array}\right] \cdot\left[\begin{array}{c}
I_{A}(t) \\
\omega(t)
\end{array}\right] } \\
& +\left[\begin{array}{cc}
\frac{1}{L_{A}} & 0 \\
0 & -\frac{1}{J}
\end{array}\right] \cdot\left[\begin{array}{c}
V_{A}(t) \\
T_{L}(t)
\end{array}\right]
\end{aligned}
$$

The armature current $I_{A}$ and the motor's rotation speed $\omega$ are the states of the differential equation system. Inputs are the armature voltage $V_{A}$ and the load torque $T_{L} \cdot R_{A}, L_{A}$ and $K_{T}$ describe the armature resistance, the phase inductivity and the engine torque constant.

3) Pump: By magnetic coupling the motor drives a centrifugal pump. Following [9] again, the pump can be modeled as

$$
\Delta p(t)=h_{N N} \omega(t)^{2}-h_{N V} \omega(t) q(t)-h_{V V} q^{2}
$$

$\Delta p(t)$ represents the pressure between pump inlet and pump outlet. $q(t)$ stands for the produced pump flow. $h_{N N}, h_{N V}$ and $h_{V V}$ are pump constants that can be identified experimentally.

4) Patient Model: Our setup for the cardiovascular component follows the Westerhof model [1]. The systemic resistance is frequency-dependent. Arteries are modeled as elastic chambers, veins and capillaries as linear resistances. The electrical analogon is a parallel circuit of a capacitor and a resistance (see Figure 2). Applying Kirchhoff's circuit laws

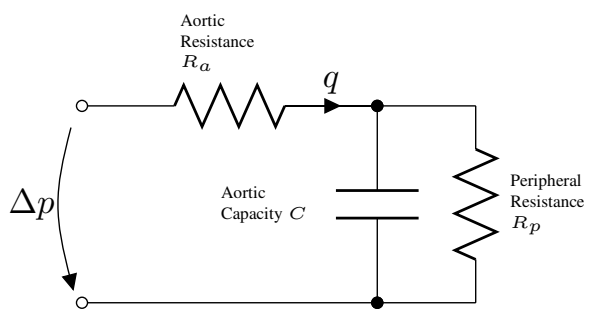

Fig. 2. Network representation of the 3-Element-Windkessel Model

the pressure-flow relationship in the CVS can be described by the following equation:

$$
\left(1+\frac{R_{a}}{R_{p}}\right) q(t)+C R_{a} \frac{d q(t)}{d t}=\frac{p(t)}{R_{p}}+C \frac{d p(t)}{d t}
$$

$R_{a}$ and $C$ describe the aortic resistance and capacity, while $R_{p}$ models the peripheral resistance. In the physical setup a change of resistances is realized by a change of the tube diameter. Resistances can be changed via step motors. Changes in the capacity are related to changes in the gas volume within a closed air chamber. They can be realized by changing the filling level of the fluid.

5) Model Aggregation and Validation: Combining (1) (3) a state space representation for pressure and flow control can be derived. E.g. using the flow as the control variable, the model can be analytically expressed as

$$
\begin{aligned}
{\left[\begin{array}{c}
\dot{\omega} \\
\dot{q}
\end{array}\right] } & =\left[\begin{array}{l}
f_{1}\left(\omega, q, V_{A}\right) \\
f_{2}\left(\omega, q, V_{A}\right)
\end{array}\right] \\
y & =q .
\end{aligned}
$$

A linearized version of this representation can be written as a singular transfer function in the frequency domain:

$$
G(s)=\frac{b_{1} s+b_{0}}{s^{2}+a_{1} s+a_{0}}
$$

This transfer function is the basis for the controller design described in the following subsections.

To validate our model, we measure flow and pressure under step changes of the input variable $V_{A}$ and compare the results with the predicted values. Figure 3 shows the dynamics of the motor speed, the flow and the pressure. We observe small but still acceptable deviations between the measured and simulated dynamics. Those model uncertainties are accounted for in the controller design if possible. The controllers are optimized for a minimal integral of absolute error (IAE) in the step response of the nominal system.
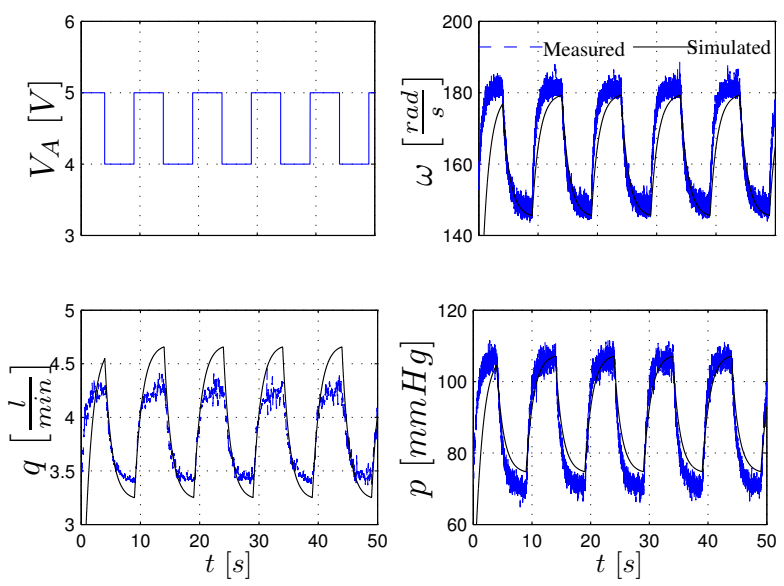

Fig. 3. Comparison of simulation model with hydraulic model. Motor speed (top right), flow (bottom left) and pressure (bottom right) characteristics as a response to step changes in the input signal (top left).

\section{B. Proportional-Integral Controller}

The Proportional-Integral Controller (PI) is one of the most established methodologies in control theory. Its mechanism is rather simple. The P-Contribution accounts for the present error while the I-Contribution accounts for accumulated errors in the past. The control output $u(t)$ in the time domain is given as

$$
u(t)=K_{P} e(t)+K_{I} \int_{0}^{t} e(\tau) \mathrm{d} \tau .
$$

The parameter $K_{P}$ proportionally weights the offset $e(t)$, while $K_{I}$ amplifies the integrated control deviation. Following the Parameter Space Approach [10], we identify suitable values for $K_{P}$ and $K_{I}$. To robustly design the PI Controller for the mock system we varied the motor's inertia $J$, the friction torque $T_{F l}$ and the pump constants by $10 \%$. From all candidates for stable control we select a $K_{P}-K_{I}$-pair that reveals minimal overshoot and oscillations $\left(K_{P}=1.6\right.$, $\left.K_{I}=1.5\right)$. 


\section{C. $H_{\infty}$-Controller}

The $H_{\infty}$-Approach is a robust control strategy. It is based on frequency response characteristics that result in a controller that accounts for particular specifications such as bandwidth limitations or robustness to parameter uncertainties [11]. The standard $S / K S / T$ scheme is depicted in Figure 4 . The weighting functions $W_{S}, W_{K S}$ and $W_{T}$ can be used to influence the control characteristics. In Figure 5(a)

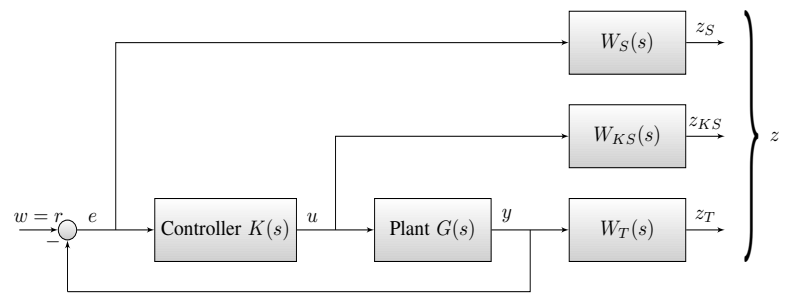

Fig. 4. Standard $\mathrm{H}-\infty$ Weighting Scheme.

the frequency characteristics for the nominal system (green) and the system under parameter variations (blue) are shown. Since $W_{T}$ is used to account for parameter uncertainties it is chosen such that the system weighted with $W_{T}$ covers all system variations (Figure 5(a), red). Figure 5(b) shows the selected amplitude response of $W_{S}$ and $W_{T}$. We choose $W_{K S}$ small and constant $\left(W_{K S}=1 e^{-4}\right)$.

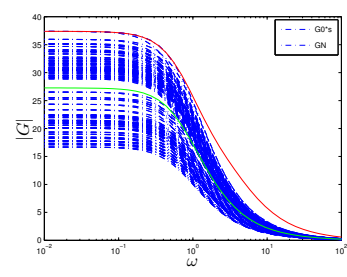

(a)

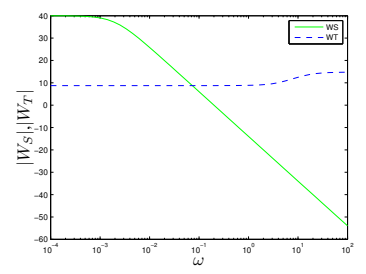

(b)
Fig. 5. Amplitude response of the nominal system (green), under parameter variations (blue), and the $W_{T}$-weighted nominal system (red) (a). Amplitude response of $W_{S}$ and $W_{T}(\mathrm{~b})$.

\section{PI-Fuzzy Controller}

Fuzzy control is an established method in control engineering and has been applied to a wide range of problems. No analytical plant description is needed. Also the fuzzy inference logic allows to interpret linguistic rules to design controllers based on knowledge from domain experts.

In this work we use a PI-Fuzzy Controller based on the work of $\mathrm{Li}$ and Gatland [12]. Two input variables are used: the control error $e$ and its derivative. The output variable controls the increase or decrease of the pump speed. The membership functions for the two inputs and the output are composed of 7 sets each. The rules were created as described in [12]. Internally the Fuzzy-PI works in the range of -1 to 1 and gains are used to transfer inputs and outputs to the internal representation. We tuned the controller by a grid search over the gain parameters. For flow control we set the proportional gain to $K_{p}=0.225$ and the derivative gain to $K_{d}=0.1$. The output gain was set to $K_{i}=4$. For pressure control the parameters were chosen as $K_{p}=K_{d}=0.05$ and $K_{i}=4$. Figure 6 shows the schematic control loop.

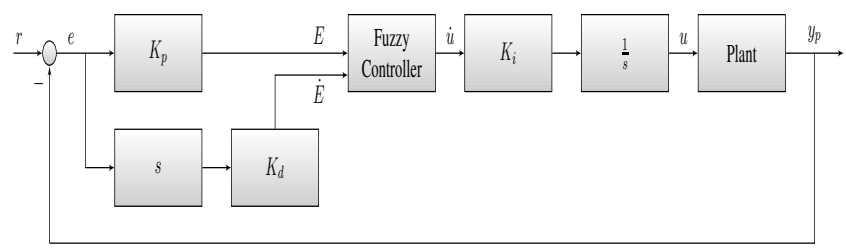

Fig. 6. PI-Fuzzy control loop.

\section{E. Model Reference Adaptive Controller}

Model Reference Adaptive Control (MRAC) is one of the main approaches to Adaptive Control. A reference model is chosen to generate a desired trajectory $y_{m}$ that the plant output $y_{p}$ has to follow. The tracking error $e=y_{p}-y_{m}$ represents the deviation of the plant output from the desired trajectory [13]. Based on the error and the control and plant output the controller is redesigned. The MRAC feedback loop is shown in Figure 7. We apply a control law and

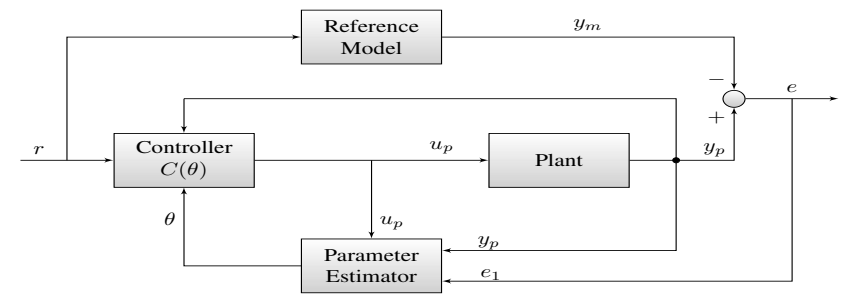

Fig. 7. MRAC loop.

a parameter estimation mechanism for second-order SISOSystems with a relative degree of $n^{*}=1$ as derived in [13]: We chose the reference model as

$$
W_{m}(s)=\frac{3}{s+3} .
$$

The control law reads

$$
\begin{aligned}
& \dot{\omega}_{1}=-2 \omega_{1}+u_{P}, \quad \omega_{1}(0)=0 \\
& \dot{\omega}_{2}=-2 \omega_{2}+y_{P}, \quad \omega_{2}(0)=0 \\
& u_{p}=\theta_{1} \omega_{1}+\theta_{2} \omega_{2}+\theta_{3} y_{P}+c_{0} r .
\end{aligned}
$$

The control parameters that are to be identified by the parameter estimator can be summarized in the vector $\theta=$ $\left[\theta_{1} \theta_{2} \theta_{3} c_{0}\right]^{T}$. Together with $\left[\omega_{1} \omega_{2} y_{P} c_{0} r\right]^{T}$ and $e$ they can be calculated using a gradient descent procedure

$$
\dot{\theta}_{i}=-\gamma_{i} e \omega_{i}, \quad i=1, \ldots, 4
$$

with step size $\gamma_{i}$. 


\section{F. Evaluation}

We evaluate and compare the control strategies in two scenarios. First we change the control target. For pressure control we switch between 40,60 and $80 \mathrm{mmHg}$. For flow control we change the targets to 3,4 and $51 / \mathrm{min}$. In the second scenario we vary the peripheral resistance. We vary it fast (1 second to target) and slow (10 seconds). As an error measure we use the IAE:

$$
e=\int_{t=0}^{T}|r(t)-y(t)| d t
$$

\section{RESULTS AND DISCUSSION}

Table I shows the IAE for changes in the target values during flow control. Figure 8 visualizes the controllers' response to step changes. The PI controller reaches the target values fast and without overshoots or oscillations. For the MRAC small overshoots can be observed. The $H_{\infty}$-Controller shows smooth behavior but does not follow changes of the target value as quick as the PI. The PI-Fuzzy Controller was also able to follow the step changes, but only with overshoots and oscillations.

TABLE I

IAE FOR STEP CHANGES OF THE CONTROL TARGET

\begin{tabular}{|l||c|c|c|c|}
\hline & PI & $H_{\infty}$ & MRAC & Fuzzy \\
\hline Flow control & 7.9 & 14.5 & 13.8 & 15.8 \\
\hline Pressure control & 140.8 & 213.5 & 207.6 & 309.9 \\
\hline
\end{tabular}

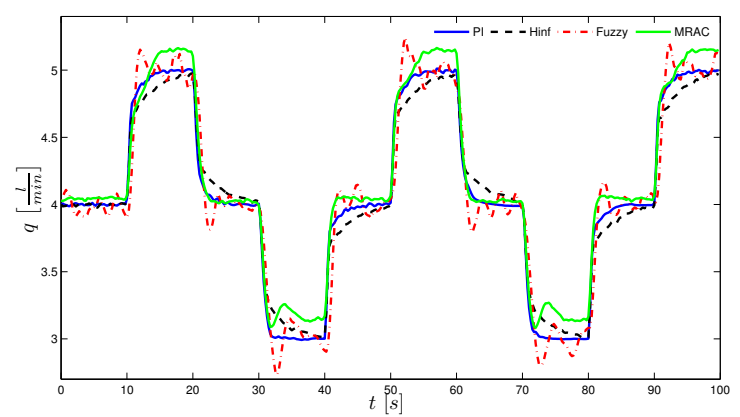

Fig. 8. Step response of control strategies during flow control.

In our second scenario (change of peripheral resistance) these results were fostered. Again the PI was ranked first. However the $H_{\infty}$-Controller is outperformed by the MRAC. This can be explained by the adaptive capabilities of the MRAC.

In all our experiments (also in pressure control) the PI controller revealed the best performance. In terms of IAE it outperformed its competitors, showed smooth behavior and reacted fast to parameter changes. For the control design an analytical system model is needed. The controller can be designed to account for parameter uncertainties. The $H_{\infty^{-}}$ Controller also showed smooth but sometimes inert behavior. Again a system model is needed and model uncertainties can be considered in the design process. The MRAC's behavior depends on the parameter estimation process. Generally rich input signals (pulsatile) are favorable for a good identification. Constant inputs lead to bad identification which is the reason why sometimes the targets are not reached. The MRAC does not need a complete system model but the system's order is sufficient. This is also the main advantage of the Fuzzy Controller. Since the design is knowledge-based no analytical model is needed. However, target values were not reached exactly and oscillations were observed.

\section{CONCLUSION AND FUTURE WORKS}

In this work we presented a layout of a 3-element windkessel model. We derived an analytical description and implemented 4 control strategies for flow and pressure control under extra-corporal circulation. Quantitatively the PI controller gave the best results.

From a physiological point of view fast reactions with small control errors (such as for the PI controller) might not be as favorable as a smooth but slightly inert control (e.g. $H_{\infty^{-}}$ Control). However this has to be discussed in the medical community.

\section{ACKNOWLEDGMENTS}

This work was supported by the Graduate School of Information Science in Health (GSISH) and an unrestricted grant from Bayerische Forschungsstiftung.

\section{REFERENCES}

[1] N. Westerhof, G. Elzinga, and P. Sipkema, "An artificial arterial system for pumping hearts," Journal of Applied Physiology, vol. 31, no. 5, pp. 776-781, Nov. 1971.

[2] N. Stergiopulos, B. E. Westerhof, and N. Westerhof, "Total arterial inertance as the fourth element of the windkessel model," American Journal of Physiology - Heart and Circulatory Physiology, vol. 276, no. 1, pp. H81-H88, Jan. 1999.

[3] T. Kind, T. Faes, J.-W. Lankhaar, A. Vonk-Noordegraaf, and M. Verhaegen, "Estimation of three- and four-element windkessel parameters using subspace model identification," Biomedical Engineering, IEEE Transactions on, vol. 57, no. 7, pp. 1531-1538, 2010.

[4] M. Sharp and R. Dharmalingam, "Development of a hydraulic model of the human systemic circulation," ASAIO, vol. 45, no. 6, pp. 535$540,1999$.

[5] V. Prilutskii, S. Tropskii, V. Khrenov, and Y. Slavyak, "Analysis of control systems for extracorporeal-circulation equipment," Biomedical Engineering, vol. 12, no. 4, pp. 196-199, Jul. 1978. [Online]. Available: http://dx.doi.org/10.1007/BF00562045

[6] R. R. Rao, B. Aufderheide, and B. W. Bequette, "Experimental studies on multiple-model predictive control for automated regulation of hemodynamic variables," IEEE Transactions on Biomedical Engineering, vol. 50, pp. 277-288, 2003.

[7] B. Misgeld, J. Werner, and M. Hexamer, "Robust and self-tuning blood flow control during extracorporeal circulation in the presence of system parameter uncertainties," Medical and Biological Engineering and Computing, vol. 43, no. 5, pp. 589-598, Oct. 2005.

[8] G. Meyrowitz, "Automatisierung der herz-lungen-maschine," Ph.D. dissertation, Universität Karlsruhe, 2005.

[9] R. Isermann, Mechatronic Systems Fundamentals. Springer, 2005.

[10] J. Ackermann, Robust Control: The Parameter Space Approach. Springer, 2002.

[11] H. Geering and C. Roduner, "Entwurf Robuster Regler mit der $H_{\infty^{-}}$ Methode," Bulletin SEV/VSE, vol. 3, pp. 55-58, 1999.

[12] H.-X. Li and H. Gatland, "Conventional fuzzy control and its enhancement," IEEE Transactions on Systems, Man, and Cybernetics, vol. $26,5,1996$.

[13] P. Ioannou, Robust Adaptive Control. Prentice Hall, 1995. 